ECCOMAS

Proceedia
COMPDYN 2021

$8^{\text {th }}$ ECCOMAS Thematic Conference on Computational Methods in Structural Dynamics and Earthquake Engineering M. Papadrakakis, M. Fragiadakis (eds.)

\title{
ON THE USE OF INSTANTANEOUS POWER FOR NEAR-FAULT RECORD MODIFICATION
}

\author{
Esra Zengin ${ }^{1}$, Norman A. Abrahamson ${ }^{2}$ \\ ${ }^{1}$ Beykent University Department of Civil Engineering, Istanbul, Turkey, 34398 \\ e-mail: esrazengin@gmail.com \\ ${ }^{2}$ UC Berkeley, Department of Civil and Environmental Engineering, CA, 94720 \\ e-mail: abrahamson@berkeley.edu
}

\begin{abstract}
The current seismic design and assessment procedures describe the seismic demand in terms of elastic spectral accelerations, which may not be adequate to capture the destructive effects of near-fault ground motions. The relevant characteristics of near-fault records, such as the presence of a velocity pulse, the pulse amplitude, and the pulse period $\left(T_{p}\right)$, make the record selection and modification task more challenging. A newly proposed intensity measure, called Instantaneous Power $\left(I P\left(T_{1}\right)\right)$, takes into account the effects of velocity pulses on structural response. It has been shown that vector IM including $I P\left(T_{1}\right)$ and elastic spectral acceleration at the first mode period of structure $S a\left(T_{1}\right)$ results in more reliable and accurate structural responses compared to the traditional IM, $S a\left(T_{1}\right)$. A new matching method is developed to incorporate the effects of IP into the record modification process. The procedure combines a widely used time-domain spectral matching algorithm, RspMatch, and a velocity adjustment approach to simultaneously match the target response spectrum and IP spectrum. We investigate the extent to which controlling the IP parameter in the modification process affects the nonlinear structural response by comparing the results of single-degree-of-freedom (SDOF) systems and multi-degree-of-freedom (MDOF) system, under the sets of Sa-only matched and Sa- and IP-matched records. Our findings show that the consideration of IP in the record matching can help prevent potential bias in structural response.
\end{abstract}

Keywords: Near-fault ground motions, Velocity pulses, spectral matching, Record selection and modification 


\section{INTRODUCTION}

Developing robust approaches for selecting and modifying records that can represent the critical aspects of the design motions is crucial to obtaining reliable and accurate structural response estimates. In current practice, the records having similar implicit causal parameters (e.g., magnitude, distance, site condition) with the scenario earthquake are typically scaled or modified such that their median response spectrum matches the target spectrum within the period range of interest [1]. However, selecting the records on the basis of spectral accelerations may not fully represent the damaging potential of near-fault records containing directivity pulses. For instance, characterization of the near-fault ground motions including velocity pulses requires the determination of the probability of the presence of a pulse, the period of the pulse, as well as the amplitude of the pulse. The recent study showed that the vector IM consisting of $\mathrm{Sa}\left(\mathrm{T}_{1}\right)$ and Instantaneous Power (IP $\left.\left(\mathrm{T}_{1}\right)\right)$ sufficiently and efficiently captures the destructive impact of the near-fault ground motions on structural response [2]. In order to capture the damaging characteristics of these motions, it is necessary to consider the combined effects of relevant IMs, i.e. Sa and IP, in the seismic design and assessment procedures.

One of the most widely used approaches to obtain appropriate input motions for use in response history analysis is the time-domain response spectrum matching procedure, which is also known as RspMatch [3]. This technique adds wavelet function to accelerogram so as to ensure compatibility between record spectrum and target spectrum over a specified period interval. This study presents an IP matching approach that performs modification on velocity time series to match the target IP values. The proposed matching algorithm enables near-fault records to match both the target response (Sa) spectrum and IP spectrum. The non-degrading bilinear SDOF systems of varying periods and nonlinearity levels as well as a 2-story steel frame that accounts for stiffness and strength degradation are used as case studies to evaluate the performance of the proposed matching procedure for predicting response of structures to Sa-only matched records and to Sa- and IP-matched records.

\section{INSTANTANEOUS POWER}

The IP $\left(\mathrm{T}_{1}\right)$ is defined as the maximum energy rate of the bandpass-filtered velocity time series over a short time interval given by half of the structural period (i.e. $0.5 \mathrm{~T}_{1}$ ) :

$$
I P\left(T_{1}\right)=\max \left(\frac{1}{0.5 T_{1}} \int_{t_{0}}^{t_{0}+0.5 T_{1}} V_{\text {filtered }}{ }^{2}(t) d t\right)
$$

where the IP is in units of $\mathrm{cm}^{2} / \mathrm{s}^{2}, V_{\text {filtered }}$ is the bandpass-filtered velocity of the record in $\mathrm{cm} / \mathrm{s}$. A Butterworth bandpass filter with bandpass period between $0.2 \mathrm{~T}_{1}$ and $3 \mathrm{~T}_{1}$ is applied to a velocity time series. Figure 1 illustrates the original and filtered velocity time series of the 1979 Imperial Valley-06 earthquake (Agrarias station) record. Figure 1 also shows the variation of $\operatorname{IP}\left(\mathrm{T}_{1}\right)$ level over the selected time interval $\left(0.5 \mathrm{~T}_{1}\right)$ and the Cumulative Squared Velocity (CSV) of the filtered velocity. It can be seen that the maximum IP occurs at around 10 seconds, which corresponds to the steepest slope of the CSV. The previous findings showed that the IP has a significant influence on the structural response after eliminating the effects of spectral shape and amplitude of the ground motions [2-4]. 

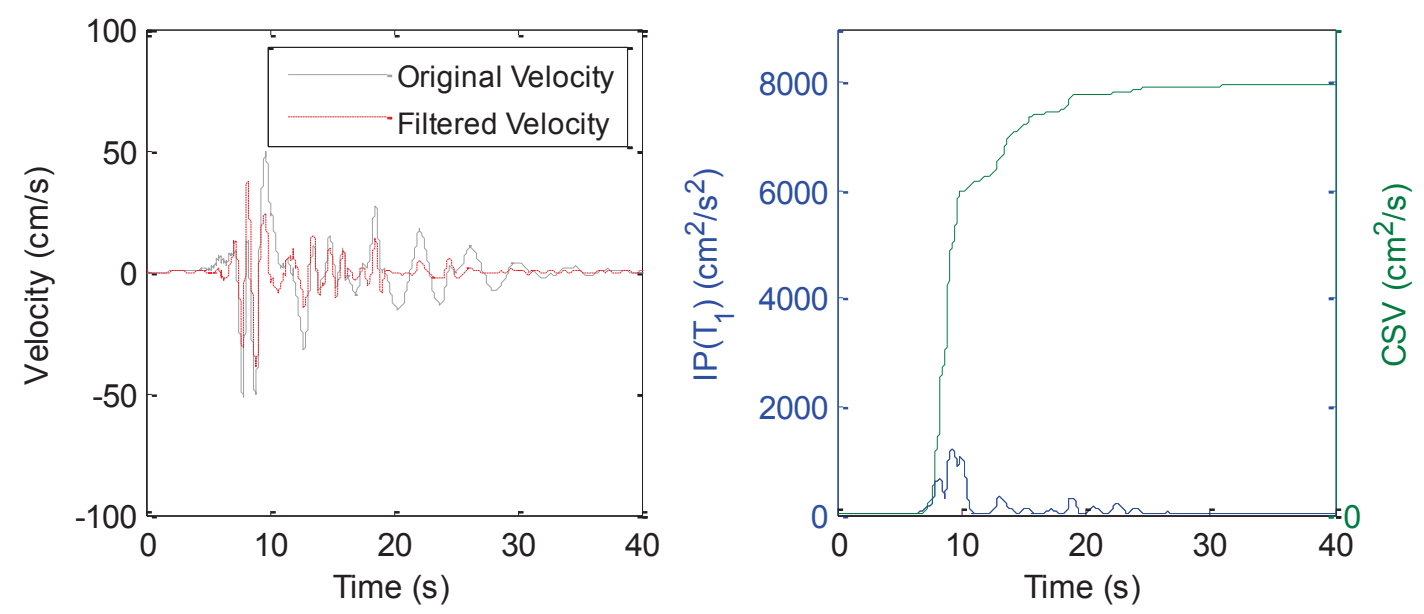

Figure 1. The original and filtered velocity time series of the 1979 Imperial Valley-06 earthquake (Agrarias station) pulse record, and the variation of $\operatorname{IP}\left(\mathrm{T}_{1}\right)$ over the selected time interval $\left(0.5 \mathrm{~T}_{1}\right)$ and the Cumulative Squared Velocity (CSV) of the filtered velocity.

\section{CONSTRUCTION OF TARGET SPECTRA FOR MATCHING}

Matching of an individual component to a target median can lead to artificial suppression of the spectral variability as the target spectrum generated using geometric-mean or RotD50 (i.e., the median single-component horizontal ground motion across all nonredundant azimuth) of the ground motion does not include the peaks and troughs of the arbitrary component. To maintain the variability of the individual component of the record about the target, we construct a component-specific target spectrum, which is obtained using the ComponentVariability Ratio (CVR) given by:

$$
C V R_{i j}=\frac{S a_{i}^{H_{j, \text { record }}}}{S a_{i}^{\text {RotD50,record }}}
$$

where $S a^{\text {RotD50,record }}$ is the RotD50 spectrum of the record, and $S a_{i}^{H_{j, \text { record }}}$ corresponds to the spectral acceleration of the $j$ component of the record at the $i$ period. The component- specific target spectrum $S a_{i}^{H_{j, t a r g e t}}$ is then computed as follows:

$$
S a_{i}^{H_{j, t \text { arget }}}=C V R_{i j} \times S a_{i}^{t \operatorname{targ} e t}
$$

where $S a_{i}^{t a r g e t}$ is the target spectral accelerations. In computing the CVR for componentspecific IP target, we employ geometric-mean IP values of the record instead of the RotD50 because the conditional GMM for the IP is developed based on the geometric-mean horizontal component of ground motion [5]. A conditional ground-motion model (GMM) for $\sqrt{I P(T)}$ is given by;

$$
\ln (\sqrt{I P(T)})=a_{1}+a_{2} \ln (S a(T))+a_{3}(M-6)+a_{4} \ln \left(R_{r u p}+5 \exp (0.4(M-6))\right)+\delta B_{e}+\delta W_{e s}
$$


in which $M$ is the moment magnitude, $R_{r u p}$ is the rupture distance in kilometers, and the 5\%damped $\mathrm{Sa}$ in $\mathrm{g}$. The subscripts $e$ and $s$ correspond to the indexes of the earthquake and the site, respectively. A more detailed description of the model can be found in [5].

Figure 2 illustrates $50^{\text {th }}$ percentile (median) of the target scenario spectrum generated using the Abrahamson et. al. GMM [6], for an earthquake scenario with Magnitude $=7, \mathrm{R}_{\mathrm{rup}}=3 \mathrm{~km}$, and site condition $\mathrm{Vs}_{30}=400 \mathrm{~m} / \mathrm{s}$, target IP spectrum, as well as component-specific Sa- and IP-spectra of the selected pulse record that are used as targets in the matching.
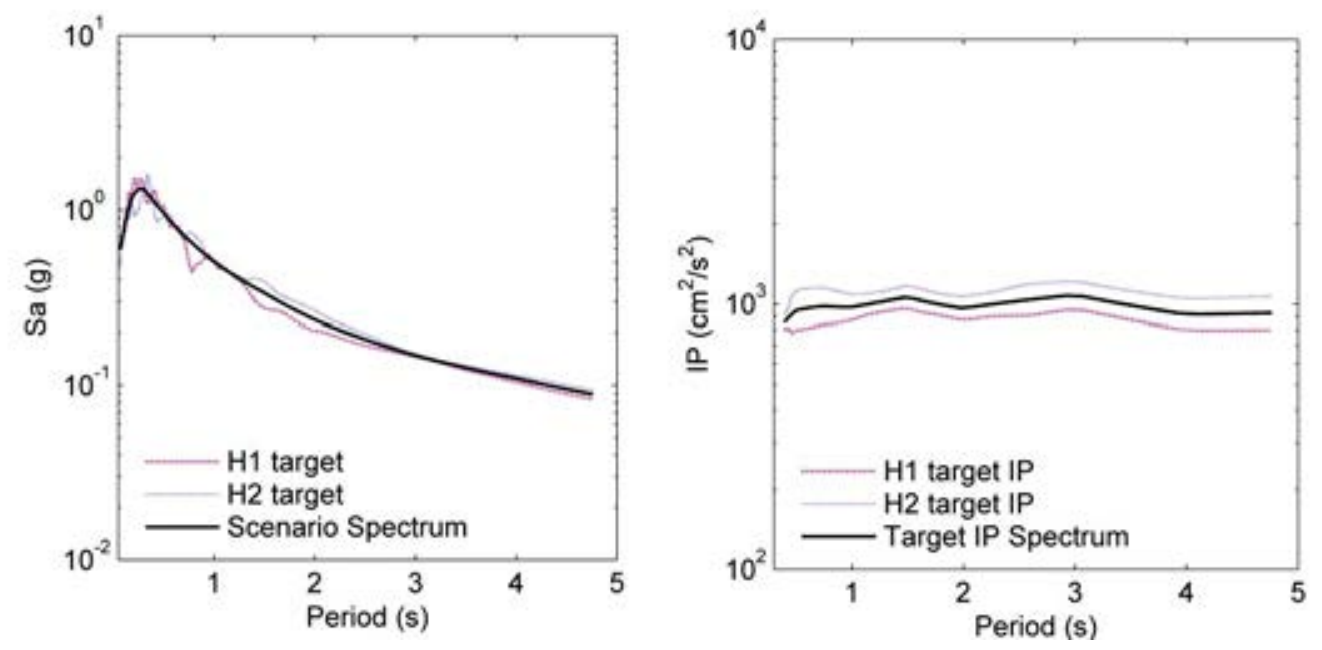

Figure 2. Comparisons of median target spectrum for an earthquake scenario with Magnitude $=7, \mathrm{R}_{\text {rup }}=3 \mathrm{~km}$, and site condition $\mathrm{Vs}_{30}=400 \mathrm{~m} / \mathrm{s}$, target IP spectrum, and component-specific Sa- and IP- spectra of the selected pulse record.

\section{IP ADJUSTMENT}

In this section, we briefly mention about the IP adjustment approach that adds a Ricker wavelet to the velocity time series so as to match the target IP values [7]. The Ricker wavelet has good time localization and a broader frequency spectrum compared to the tapered cosine function, which is added to acceleration time history in response-spectrum matching. This adjustment function is centered at the time of IP occurs $\left(t I P_{j}\right)$, for the spectral period $T_{j}$, and is given by

$$
h_{j}(t)=\left(1-2 \pi^{2} f^{2}\left(t-t I P_{j}\right)^{2}\right) \exp \left(-\pi^{2} f^{2}\left(t-t I P_{j}\right)^{2}\right)
$$

The adjusted velocity is defined as:

$$
V_{1}(t)=V_{o}(t)+\gamma_{v e l} \delta V_{1}(t)=V_{0}(t)+\sum_{j=1}^{N} b_{j} h_{j}(t)
$$

where $\delta V_{1}(t)$ is the adjustment velocity time history and $\gamma_{v e l}$ is a relaxation factor for the velocity adjustment, where $b_{j}$ is a vector of amplitudes of a set of adjustment functions, and $N$ is the total number of spectral periods. The objective is to equate the change in the IP due to the adjustment to the misfit between target IP and record IP. We refer the reader to the paper by Zengin and Abrahamson [8] for more details. 


\section{MAIN STEPS OF THE SIMULTANEOUS SA- AND IP-MATCHING}

The proposed approach sequentially modifies time series to have a close match between records' spectra and target spectra. The flowchart of the proposed algorithm is shown in Figure 3. The required user inputs are target response spectrum, two horizontal components of the ground motion, time step, damping ratio, target peak ground acceleration (PGA). In the first step, the original acceleration time history is initially scaled to the target PGA of the record so as to preserve the shape of the envelope of the time series at high frequencies. The component-specific Sa- and IP- target spectra are then constructed using Equation 3. We subdivide the target periods into smaller subgroups to increase the stability of the matrix inversion as well as to avoid numerical convergence problems. In that regard, we divide Sa- and IP- periods into $k$ subgroups and select every $k^{\text {th }}$ element using the equation $k=(N / n)$, where $N$ is the total population size, and $n$ is the sample size. The maximum sample size is set to 20 because it yields optimum convergence. Moreover, we improve the stability of the matrix inversion by using a Tikhonov regularization method, which is also known as ridge regression [9].

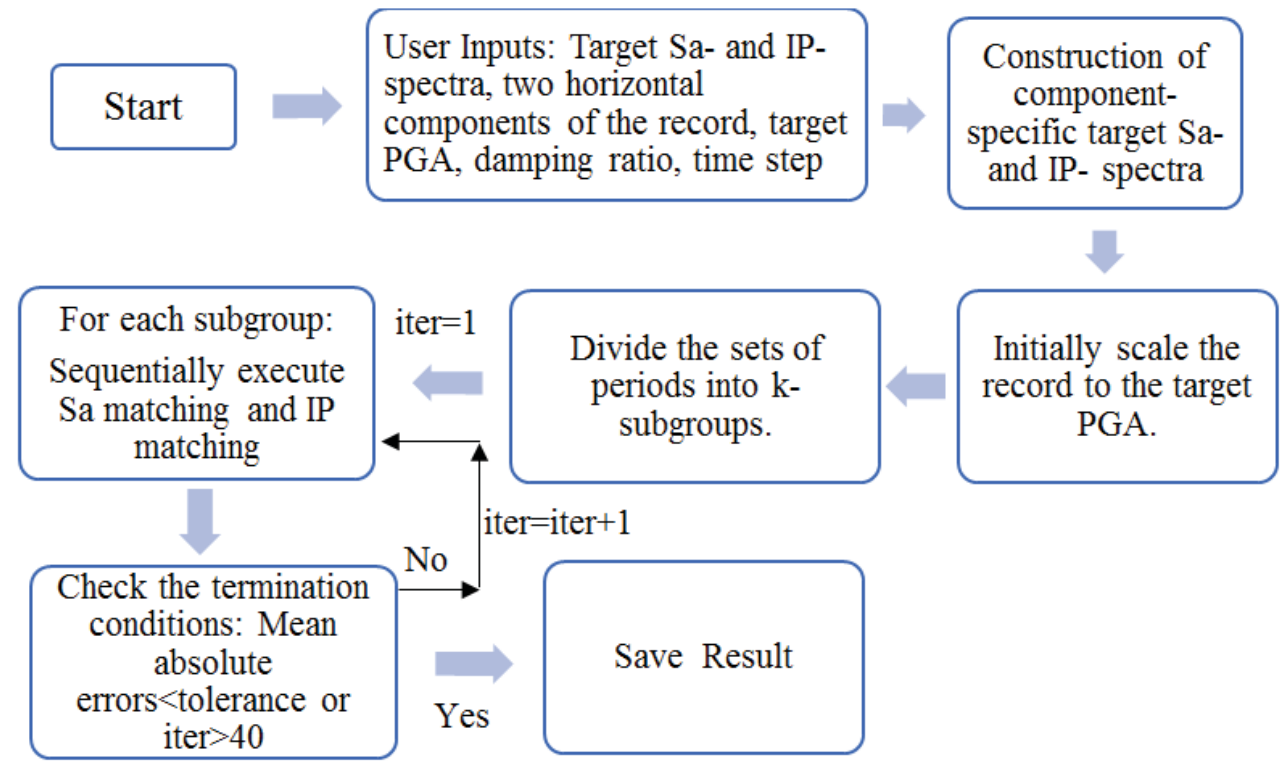

Figure 3. Flowchart of the Sa- and IP-matching algorithm.

Each iteration sequentially performs Sa matching and IP matching stages for each subset. In other words, the accelerogram adjusted using Sa-matching phase is integrated to obtain the velocity time history, which is then used as an input for IP matching. We note that the relaxation factors used to damp the total acceleration and velocity adjustments affect the convergence. Thus, after several trials, we select acceleration relaxation factor of 0.50 and velocity relaxation factor of 0.10 . The iterations continue until the desired tolerance levels are achieved, or the maximum number of iterations is reached. Mean Absolute Error (MAE) of the misfit between target spectrum and record spectrum over $\mathrm{N}$ periods is calculated using the following formula: 


$$
M A E=\frac{1}{N} \sum_{i=0}^{N}\left|\ln \left(y_{i}\right)-\ln \left(x_{i}\right)\right|
$$

where $y$ denotes the target value and $x$ is the current value of the reference time series. The tolerance levels for the Sa- and the IP- matching are 0.05 and 0.10 , respectively. The maximum allowable number of iterations is selected as 40 .

\section{CASE STUDY FOR RECORD MATCHING}

Figure 4 shows the acceleration, velocity, and displacement time histories of $\mathrm{H} 2$ horizontal component of the pulse record (1979 Imperial Valley-06 earthquake Agrarias station) before and after Sa- and IP- matching. The left panel of the figure illustrates the time histories of the original record, whereas the right panel shows the adjusted time series. It is seen that the addition of wavelets results in some changes in the amplitude and waveforms of the records. However, we assess the suitability of the records for nonlinear response history analysis based on Sa and IP IMs, which are pertinent to the structural response. It should be noted that the consideration of IP in the matching process alleviates the need to preserve the pulse characteristics (i.e., pulse amplitude and pulse period) in the matched record as the IP accounts for the destructive effects of the near-fault ground motion with or without velocity pulses. Thus, we do not check whether the resulting record exhibits velocity pulse.

Figure 5 compares the Sa- and IP spectra of the record and target spectra. Also plotted are the linearly scaled spectra of the record. These results indicate that the proposed matching algorithm performs well in the simultaneous matching of the Sa and IP.
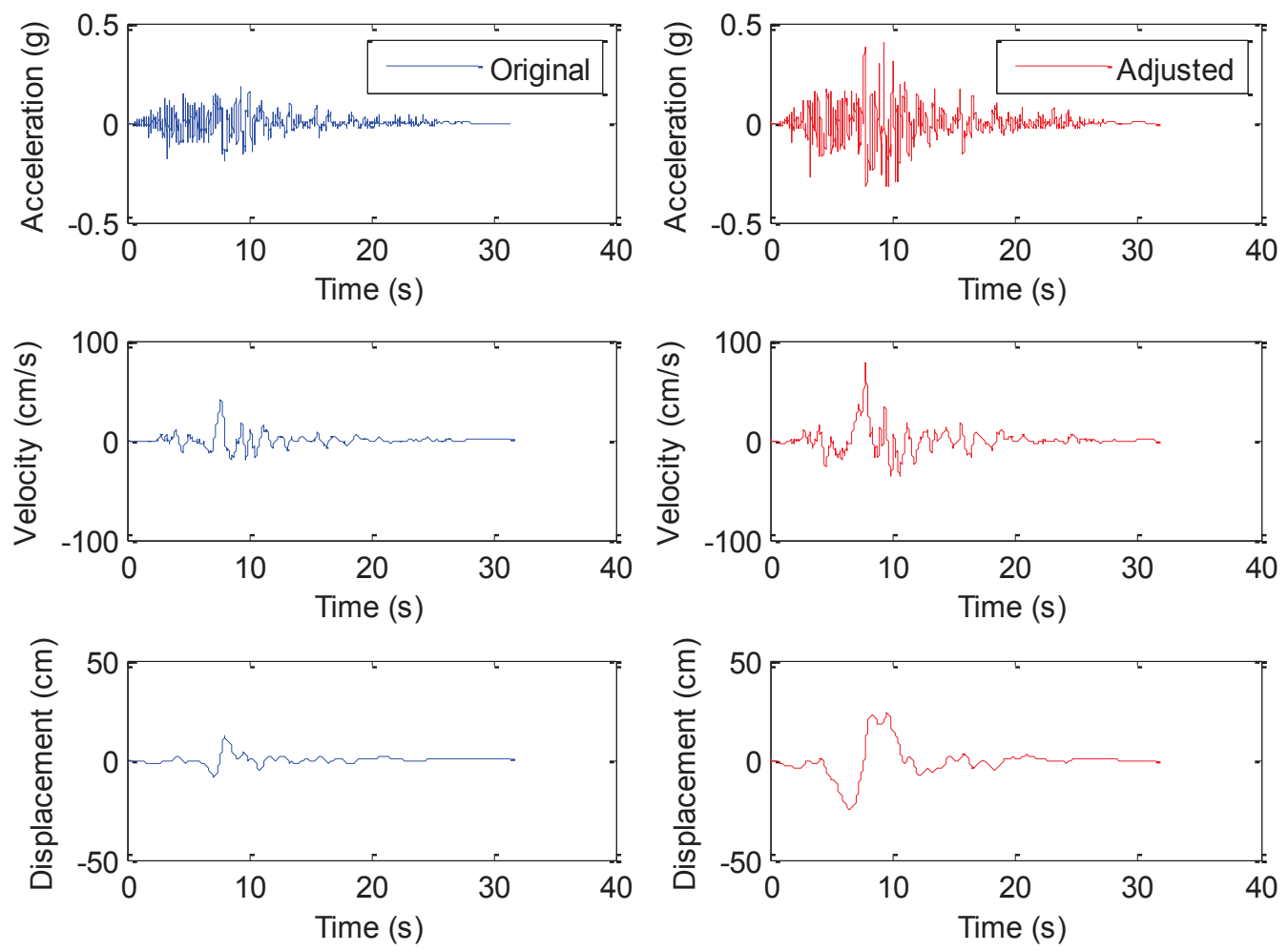

Figure 4. Acceleration, velocity, and displacement time histories of H2 component of the pulse record (1979 Imperial Valley-06 earthquake Agrarias station record) before and after Sa- and IP- matching. 

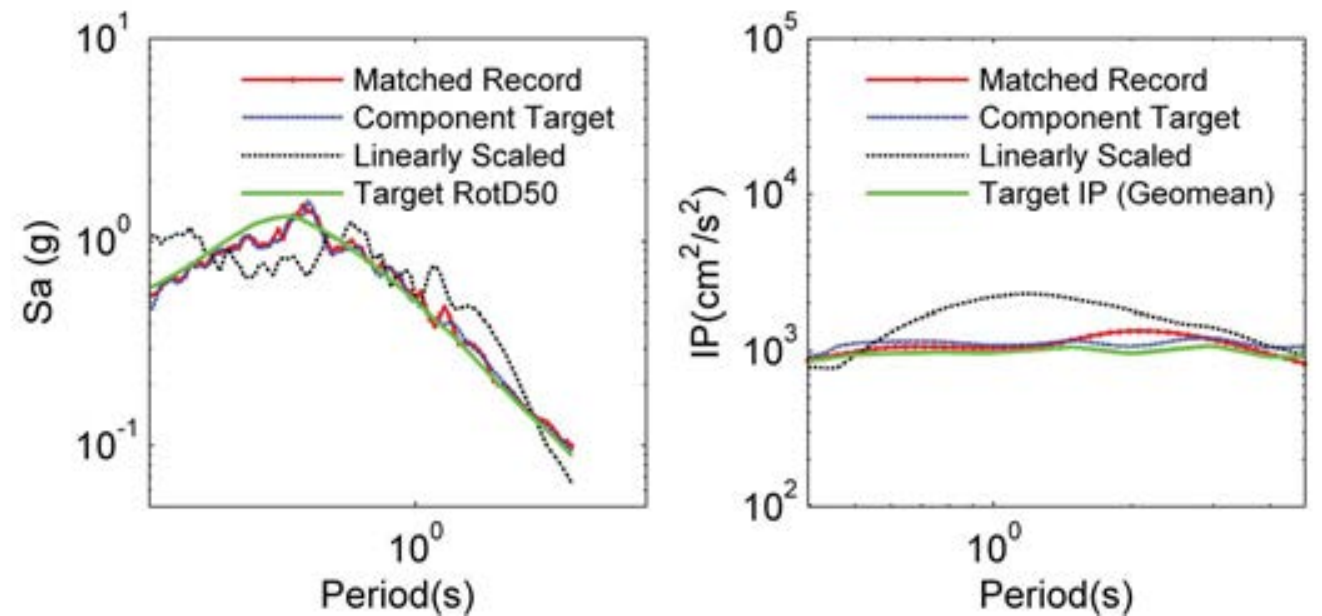

Figure 5. Comparisons of record Sa- and IP-spectra and target spectra

\section{NONLINEAR STRUCTURAL RESPONSES}

Using the proposed matching algorithm, we match 11 near-fault records to the target median scenario spectrum for an earthquake scenario with Magnitude $=7, R_{\text {rup }}=3 \mathrm{~km}$ and site condition $\mathrm{Vs}_{30}=400 \mathrm{~m} / \mathrm{s}$. In our analysis, we utilize both Sa- matched and Sa- and IP-matched records. The records are randomly selected in the magnitude range 6.5-7.5, $\mathrm{R}_{\text {rup }}$ range 0-20 $\mathrm{km}$, and $\mathrm{Vs}_{30}$ range 180-760 m/s. We perform nonlinear dynamic analyses of bilinear SDOF systems having five different structural periods between 0.6 and 3.0 seconds and two different inelasticity levels defined by strength reduction factors of $\mathrm{R}=2$ (nearly elastic) and $\mathrm{R}=6$ (highly inelastic). Damping ratio and strain hardening ratio are taken as $5 \%$ and $\alpha=0.10$, respectively. We also consider a 2-story moment-resisting frame that includes the Modified Ibarra Krawinkler stiffness and strength degradation material model [10][11]. The analytical model also takes into account the P-delta effects. The fundamental period of the frame structure is found to be $0.82 \mathrm{sec}$. The detail information about this structure can be found in [12]. In this case, the selected records are matched to the $84^{\text {th }}$ percentile of the target spectrum to obtain post-elastic maximum interstory drift ratios (MIDRs) of the structure.

Figure 6 plots the mean ratio of the IPs obtained from Sa-matched records to the IPs obtained from Sa- and IP-matched records versus period. It is seen that the mean IP value of the Sa- matched records is lower than that of the Sa- and IP-matched records. Because there is almost no difference between the Sa-values of both record sets, we may attribute the differences observed in structural response to differences in IPs.

Figure 7 shows the results of inelastic displacements of SDOF systems having various periods for $\mathrm{R}=2$ and $\mathrm{R}=6$. We see that the $\mathrm{Sa}$-only matched records produce a lower mean structural response compared to the Sa- and IP-matched records, suggesting that the effects of IP on structural response are more pronounced for the highly inelastic case. We also observe that, in most cases, the Sa- and IP-matched records produce lower levels of dispersion compared to Sa-only matched records. Figure 8 shows the MIDRs of a 2-story steel frame structure obtained using Sa- matched and Sa- and IP-matched records, where dashed lines show the median results of the record sets. As seen, the MIDRs of Sa-matched records are, on average, lower than that of Sa- and IP -matched records. This indicates that the records having lower IP values than the target IP can produce biased-low responses, relative to the Sa- and IP-matched 
record, highlighting the importance of considering the IP parameter in the record modification process.

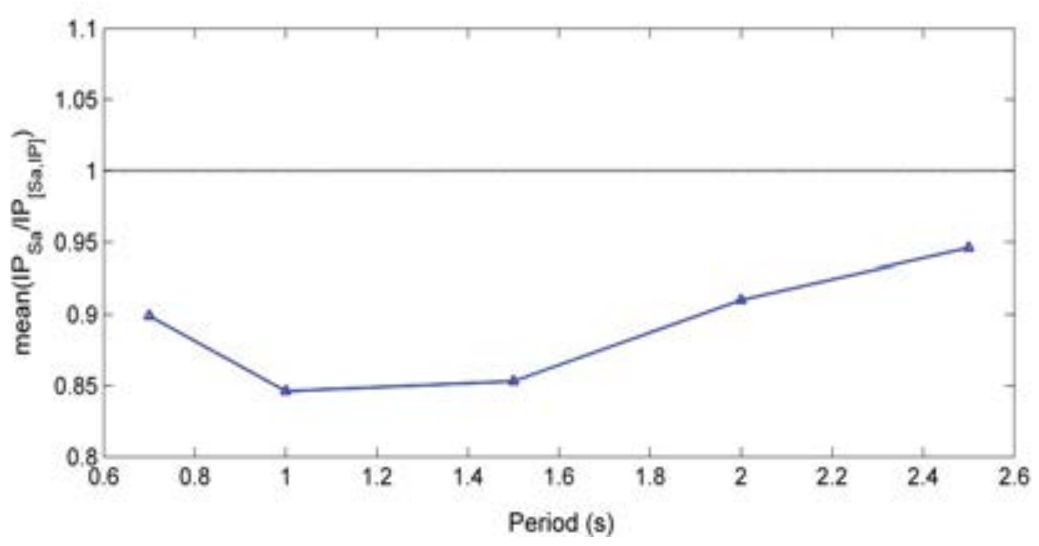

Figure 6. Mean ratio of the IPs obtained from Sa-matched records to the IPs obtained from Sa- and IP-matched records.
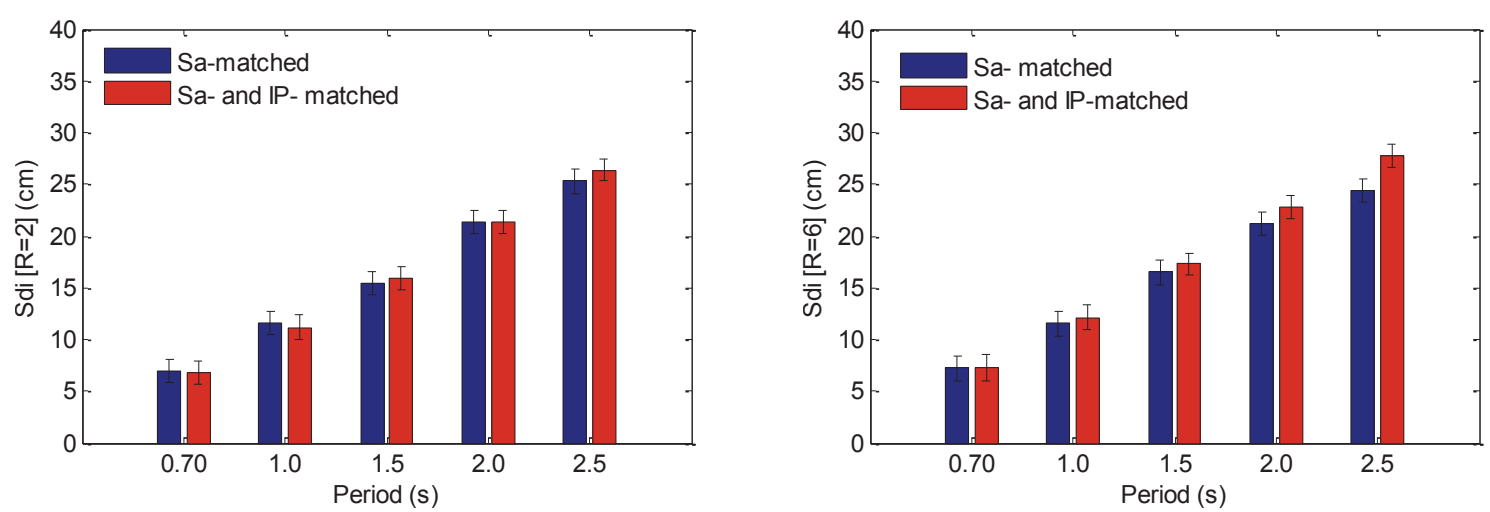

Figure 7. Inelastic displacements of SDOF systems having various periods for $\mathrm{R}=2$ and $\mathrm{R}=6$.

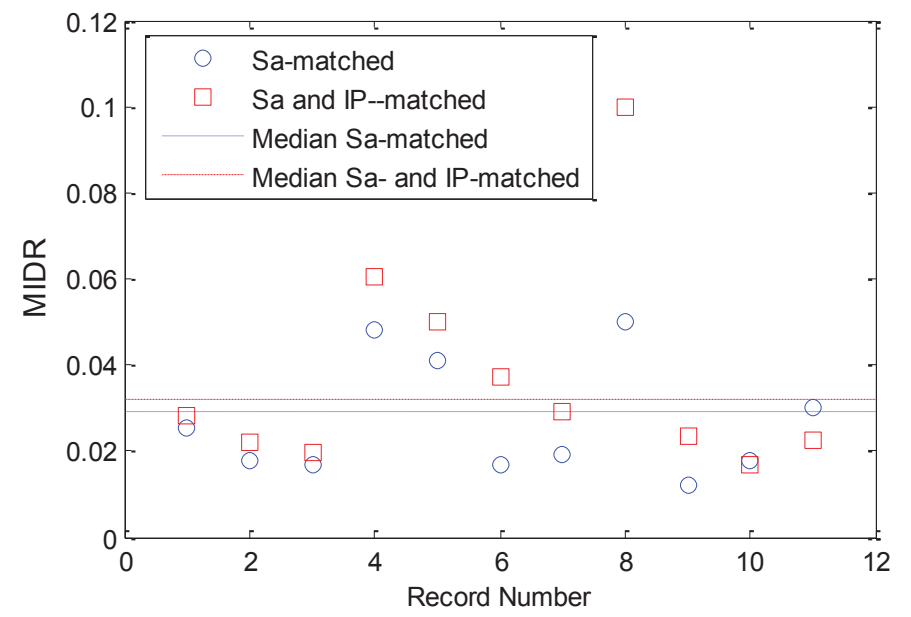

Figure 8. MIDRs of 2-story steel frame $\left(\mathrm{T}_{1}=0.82 \mathrm{~s}\right)$ obtained using Sa- matched and Sa- and IP-matched records. 


\section{CONCLUSIONS}

This paper proposes a ground-motion matching procedure that adjusts the near-fault records to simultaneously match target Sa- and IP- spectra. The algorithm builds upon the existing technique, RspMatch, for spectral matching and extends the time-domain modification for IP matching. The case study results show that our algorithm can effectively match the target values over a range of periods. One advantage of considering IP IM is that there is no need to preserve the pulse characteristics in the matching process because IP takes into account the effects of velocity pulses (i.e., pulse amplitude and pulse period). The results of this study indicate that the proposed matching approach can prevent potential bias in nonlinear structural response.

The Matlab source code and the documentation for the Sa- and IP- matching procedure are available at (https://github.com/zenginesra/Sa_IPmatch).

\section{REFERENCES}

[1] ASCE, Minimum Design Loads for Buildings and Other Structures, ASCE/SEI 7-16, American Society of Civil Engineers, Washington, D.C., 2016.

[2] Zengin, E., N. A. Abrahamson, A vector-valued intensity measure for near-fault ground motions, Earthquake Engineering and Structural Dynamics, 49.7, 716-734, 2020.

[3] Abrahamson, N. A, Non-stationary spectral matching, Seismological research letters, 63 (1), 30, 1992.

[4] Zengin, E., N. A. Abrahamson, S. Kunnath, Isolating the effect of ground-motion duration on structural damage and collapse of steel frame buildings, Earthquake Spectra, 36 (2), 718-740, 2020.

[5] Zengin, E., N. Abrahamson, Conditional ground-motion model for damaging characteristics of near-fault ground motions based on instantaneous power, Bulletin of the Seismological Society of America, 110 (6), 2828-2842, 2020.

[6] Abrahamson, N. A., W. J. Silva, R. Kamai, Summary of the ASK14 ground motion relation for active crustal regions, Earthquake Spectra, 30(3), 1025-1055, 2014.

[7] Ricker, N., The form and laws of propagation of seismic wavelets, Geophysics, 18 (1), 10-40, 1953.

[8] Zengin, E., N. A. Abrahamson, A Procedure for matching the near-fault ground motions based on spectral accelerations and instantaneous power, Earthquake Spectra, in print, 2021.

[9] Hoerl, A.E., R.W. Kennard, Ridge regression: Biased estimation for nonorthogonal problems, Technometrics, $12.1: 55-67,1970$.

[10] Ibarra, L.F., R.A. Medina, H. Krawinkler, Hysteretic models that incorporate strength and stiffness deterioration, Earthquake Engineering and Structural Dynamics, 34, 1489$1511,2005$.

[11] Lignos, D., Sidesway collapse of deteriorating structural systems under seismic excitations, Ph.D. Thesis, Stanford University,2008.

[12] Eads, L., Dynamic Anaysis of 2-Story Moment Frame, https://opensees.berkeley.edu/wiki/index.php/Dynamic_Analysis_of_2-

Story_Moment_Frame, last accessed April 2021. 